\title{
Numerical Solutions of Linear Fractional Order BVP by Galerkin Residual Method with Differentiable Polynomials
}

\author{
Umme Ruman ${ }^{1}$, Md. Shafiqul Islam ${ }^{2, *}$ \\ ${ }^{1}$ Department of Computer Science \& Engineering, Green University of Bangladesh, Dhaka, Bangladesh \\ ${ }^{2}$ Department of Applied Mathematics, University of Dhaka, Dhaka, Bangladesh
}

Email address:

ruman@cse.green.edu.bd (U. Ruman), mdshafiqul@du.ac.bd (Md. S. Islam)

${ }^{*}$ Corresponding author

To cite this article:

Umme Ruman, Md. Shafiqul Islam. Numerical Solutions of Linear Fractional Order BVP by Galerkin Residual Method with Differentiable Polynomials. Applied and Computational Mathematics. Vol. 9, No. 2, 2020, pp. 20-25. doi: 10.11648/j.acm.20200902.11

Received: April 9, 2020; Accepted: May 3, 2020; Published: May 14, 2020

\begin{abstract}
The main objective of this paper is to solve numerically the differential equations of fractional order with homogeneous boundary conditions by the Galerkin weighted residual method. In this method, linear combinations of some types of functions are used to find the approximate solutions which must satisfy the homogeneous boundary conditions. Such type of functions should be differentiated and integrated easily, so the piecewise polynomials, namely, Bernstein, Bernoulli and Modified Legendre polynomials are used as basis functions in this paper. The fractional derivatives are used in the conjecture of Riemann-Liouville and Caputo sense. Thus, we develop the Galerkin weighted residual formulation, in matrix form, to the linear fractional order boundary value problems, in details, which is easy to understand. The accuracy and applicability of the present method are demonstrated through few numerical examples. We observe that the approximate results converge monotonically to the exact solutions. In addition, we compare the approximate results with the exact solutions, and also with the existing solutions which are available in the literature. The absolute errors are depicted in tabular form as well as graphical representations, a reliable accuracy is achieved. The proposed method may be applied to fractional order partial differential equations also.
\end{abstract}

Keywords: Galerkin Method, Fractional Derivatives, Riemann-Liouville Derivative, Caputo Derivative, Fractional Order BVP

\section{Introduction}

In recent few decades, the wide spread of differential equations of fractional order in various forms arise in the areas of physical sciences, mathematical biology and engineering problems. These problems have been studied for existence and uniqueness results in a limited way analytically $[1,2]$ or semi-analytically $[3,4]$, such as by variational iteration and Adomian decomposition methods. For this, many researchers have attempted for solving several problems numerically, in a wide range, by different methods to obtain approximate solutions, such as Sinc-Galerkin method [5], generalized differential transform method [6], cubic spline solution [7], Cubic B-Spline wavelet collocation method [8], Legendre wavelet approximation [9], collocation-shooting method [10], and more, which are available in the literature. The technique of using the solutions of integer order differential equations has been introduced in [11] to find the exact solutions of fractional IVP, but the researchers in [12] have shown by some counter examples that it was wrong. Very recently, orthonormal fractional-order Legendre wavelet basis functions have also been incorporated in [13].

However, for the huge demand of numerical methods with great accuracy, we are interested to find the numerical solutions of linear fractional order two-point boundary value problems with homogeneous boundary conditions only. For this, in this paper, we propose Galerkin weighted residual (GWR) method [14] for approximate solutions. Here we are restricted to use the piecewise polynomials which are used extensively, differentiated and integrated easily, and may be approximated any functions to get the desired accuracy. Thus we exploit Bernstein and Modified Legendre polynomials 
[15], and also Bernoulli polynomials [16] as basis functions in the approximation.

We consider throughout the paper the most common concepts of Riemann-Liouville and the derivatives in Caputo sense which are also rewritten in section 2 as preliminaries. We use 'differ-integral' as an operator that means both derivative and integral of arbitrary order. The fractional integral is used for describing the cumulating of some quantity and the fractional derivative is used for describing damping. The Gamma function plays the important role to find the new solution of the Galerkin method.

Galerkin method to a general two-point linear boundary value problem is formulated rigorously in matrix form which is described in section 3. The proposed formulation is tested on three fractional boundary value problems in section 4 . The absolute errors, obtained by the proposed method, are displayed in tabular form. The results are also compared with the existing methods, namely, Sinc-Galerkin method [5] and Cubic- Splines method [7].

\section{Preliminaries}

Leibnitz suggested a close relationship between derivatives and infinite series, and he continued: $d^{\frac{1}{2}} x$ is equal to $x \sqrt{\frac{d x}{x}}$. In 1819 Lacorix showed that if $y=x^{a}$ then $\frac{d^{\frac{1}{2}} y}{d x^{\frac{1}{2}}}=\frac{\Gamma(a+1)}{\Gamma\left(a+\frac{1}{2}\right)} x^{a-\frac{1}{2}}$. In particular, this result obtained that $\frac{d^{\frac{1}{2}} x}{d x^{\frac{1}{2}}}=2 \sqrt{\frac{x}{\pi}}$.

In this section, firstly we show the definitions of Gamma functions and secondly Riemann-Liouville and Caputo of fractional derivatives $[1,2]$.

Gamma Function

Let $F(n)$ be a factorial function. In this case, for any positive integer $n$, given by [2]:

$$
F(0)=1, F(n)=n F(n-1) \quad n=1,2,3, \ldots
$$

Gamma function for any $x>0$ is defined as follows:

$$
\Gamma(x)=\int_{0}^{\infty} t^{x-1} e^{-t} d t .
$$

For the Gamma function can be demonstrated that:

$$
\Gamma(x+1)=x \Gamma(x) \quad x>0
$$

Also for negative values of $x$, we use the following definition:

$$
\Gamma(x)=\frac{\Gamma(x+1)}{x}
$$

Definition Let $f:[a, b] \rightarrow \mathbb{R}$ be a function, $\alpha$ be a positive real number, $n$ be the integer satisfying $n-1 \leq \alpha<n$, and $\Gamma$ be the Euler Gamma function [1], then the left and right
Riemann-Liouville fractional derivatives of order $\alpha$ of $f(x)$ are given as

$$
{ }_{a} D_{x}^{\alpha} f(x)=\frac{1}{\Gamma(n-\alpha)} \frac{d^{n}}{d x^{n}} \int_{a}^{x}(x-t)^{n-\alpha-1} f(t) d t
$$

and

$$
{ }_{x} D_{b}^{\alpha} f(x)=\frac{(-1)^{n}}{\Gamma(n-\alpha)} \frac{d^{n}}{d x^{n}} \int_{x}^{b}(t-x)^{n-\alpha-1} f(t) d t
$$

The left and right Caputo fractional derivatives of order $\alpha$ of $f(x)$ are given as

$$
{ }_{a}^{c} D_{x}^{\alpha} f(x)=\frac{1}{\Gamma(n-\alpha)} \int_{a}^{x}(x-t)^{n-\alpha-1} f^{(n)}(t) d t
$$

and

$$
{ }_{x}^{c} D_{b}^{\alpha} f(x)=\frac{1}{\Gamma(n-\alpha)} \int_{x}^{b}(-1)^{n}(t-x)^{n-\alpha-1} f^{(n)}(t) d t
$$

Bernstein Polynomials

The general form of the Bernstein polynomials of $n$th degree over the interval $[a, b]$ is defined by [15]

$$
B_{i, n}(x)=\left(\begin{array}{l}
n \\
i
\end{array}\right) \frac{(x-a)^{i}(b-x)^{n-i}}{(b-a)^{n}}, \quad a \leq x \leq b \quad i=0,1,2, \ldots, n .
$$

Bernoulli Polynomials

The Bernoulli polynomials of degree $n$ can be defined over the interval $[0,1]$ implicitly by $[16]$

$$
B_{n}(x)=\sum_{k=0}^{n}\left(\begin{array}{l}
n \\
k
\end{array}\right) b_{k} x^{n-k}
$$

where, $b_{k}$ are Bernoulli numbers given by

$$
b_{0}=1 \text { and } b_{k}=-\int_{0}^{1} B_{k}(x) d x \quad k \geq 1 .
$$

Also equations (9) can be written explicitly as

$$
\begin{aligned}
& B_{0}(x)=1 \\
& B_{m}(x)=\sum_{n=0}^{m} \frac{1}{n+1} \sum_{k=0}^{n}(-1)^{k}\left(\begin{array}{l}
n \\
k
\end{array}\right)(x+k)^{m}-\sum_{n=0}^{m} \frac{1}{n+1} \sum_{k=0}^{n}(-1)^{k}\left(\begin{array}{l}
n \\
k
\end{array}\right) k^{m}, \quad m \geq 1
\end{aligned}
$$

Modified Legendre Polynomials

The analogue of Rodrigues formula for the Legendre polynomials is

$$
p_{n}(x)=\frac{1}{n !} \frac{d^{n}}{d x^{n}}\left(x^{2}-x\right)^{n}
$$

To satisfy the condition $p_{n}(0)=p_{n}(1)=0, \quad n \geq 1$, we modify the Legendre polynomials in equation (12) as [15]:

$$
p_{n}(x)=\left[\frac{1}{n !} \frac{d^{n}}{d x^{n}}\left(x^{2}-x\right)^{n}-(-1)^{n}\right](x-1)
$$




\section{GWR Formulation of Fractional Differential Equations}

We consider Bernstein, Bernoulli and modified Legendre polynomials as basis functions to obtain the approximate solutions to the boundary value problems in the Galerkin weighted residual (GWR) method [14]. If $u(x)$ denotes the unknown exact solution to a boundary value problem then we shall denote approximate trial solution by $\tilde{u}(x)$.

We consider linear fractional order two-point boundary value problems with the boundary conditions $[1,5]$ :

$$
\frac{d}{d x}\left(p(x) \frac{d u}{d x}\right)+s(x) \frac{d^{\alpha} u}{d x^{\alpha}}=f(x), \quad u(0)=u(1)=0
$$

We assume that the approximate solution of differential equation (14) as:

$$
\tilde{u}(x)=\theta_{0}(x)+\sum_{j=1}^{n} a_{j} \theta_{j}(x)
$$

Choose $\theta_{0}(x)=0$ and $\theta_{j}(0)=\theta_{j}(1)=0 \quad$ for $\quad$ each $j=0,1, \ldots n$

Now the residual function is given by

$$
\varepsilon(x)=\frac{d}{d x}\left(p(x) \frac{d \tilde{u}}{d x}\right)+s(x) \frac{d^{\alpha} \tilde{u}}{d x^{\alpha}}-f(x)
$$

Using equations (15) and (16), the Galerkin weighted residual equations are

$$
\begin{gathered}
\int_{0}^{1} \varepsilon(x) \theta_{i}(x) d x=0 \\
\int_{0}^{1}\left[p(x) \frac{d^{2} \tilde{u}}{d x^{2}}+s(x) \frac{d^{\alpha} \tilde{u}}{d x^{\alpha}}-f(x)\right] \theta_{i}(x) d x=0 \\
\int_{0}^{1}\left[p(x) \frac{d^{2} \tilde{u}}{d x^{2}}+s(x) \frac{d^{\alpha} \tilde{u}}{d x^{\alpha}}\right] \theta_{i}(x) d x=\int_{0}^{1} f(x) \theta_{i}(x) d x
\end{gathered}
$$

Now

$$
\begin{gathered}
\int_{0}^{1} p(x) \frac{d^{2} \tilde{u}}{d x^{2}} \cdot \theta_{i}(x) d x=\left[p(x) \frac{d \tilde{u}}{d x} \cdot \theta_{i}(x)\right]_{0}^{1}-\int_{0}^{1} p(x) \cdot \frac{d \tilde{u}}{d x} \cdot \frac{d \theta_{i}}{d x} d x \\
=-\int_{0}^{1} p(x) \cdot \frac{d \tilde{u}}{d x} \cdot \frac{d \theta_{i}}{d x} d x \quad\left[\sin c e \quad \theta_{i}(1)=\theta_{i}(0)=0\right] \\
=-\int_{0}^{1} p(x)\left[\frac{d}{d x} \sum_{j=1}^{n} a_{j} \theta_{j}(x)\right] \cdot \frac{d \theta_{i}}{d x} d x \\
=-\sum_{j=1}^{n}\left[\int_{0}^{1}\left[p(x) \cdot \frac{d \theta_{j}}{d x} \cdot \frac{d \theta_{i}}{d x}\right] d x\right] a_{j}
\end{gathered}
$$

$$
\int_{0}^{1} s(x) \frac{d^{\alpha} \tilde{u}}{d x^{\alpha}} \cdot \theta_{i}(x) d x=\sum_{j=1}^{n}\left[\int_{0}^{1}\left[s(x) \frac{d^{\alpha}}{d x^{\alpha}} \theta_{j}(x)\right] \theta_{i}(x) d x\right] a_{j}
$$

Inserting equations (18) and (19) into (17) to get

$$
\left.\left.-\sum_{j=1}^{n}\left[\int_{0}^{1} p(x) \frac{d \theta_{j}}{d x} \cdot \frac{d \theta_{i}}{d x}\right] d x\right] a_{j}+\sum_{j=1}^{n}\left[s(x) \frac{d^{\alpha}}{d x^{\alpha}} \theta_{j}(x)\right] \theta_{i}(x) d x\right] a_{j}=\int_{0}^{1} f(x) \theta_{i}(x) d x
$$

or,

$$
\sum_{j=1}^{n}\left[-\int_{0}^{1}\left[P(x) \frac{d \theta_{j}}{d x} \cdot \frac{d \theta_{i}}{d x}+s(x) \frac{d^{\alpha}}{d x^{\alpha}} \theta_{j}(x) \cdot \theta_{i}(x)\right] d x\right] a_{j}=\int_{0}^{1} f(x) \theta_{i}(x) d x
$$

or, equivalently

$$
\sum_{j=1}^{n} k_{i, j} a_{j}=F_{i}
$$

where

$$
\begin{gathered}
k_{i, j}=-\int_{0}^{1}\left[p(x) \frac{d \theta_{j}}{d x} \cdot \frac{d \theta_{i}}{d x}+s(x) \frac{d^{\alpha}}{d x^{\alpha}} \theta_{j}(x) \cdot \theta_{i}(x)\right] d x \\
\text { and } \quad F_{i}=\int_{0}^{1} f(x) \theta_{i}(x) d x
\end{gathered}
$$

Solving the system (20) we find the values of parameters and then substituting into equation (15), we get the approximate solution of the desired FBVP (14).

\section{Numerical Examples}

In this section, we consider three problems with homogeneous boundary conditions to verify the proposed method.

Example 1. Consider the linear fractional boundary value problem [5]

$$
u^{\prime \prime}+{ }_{0}^{c} D_{x}^{0.5} u(x)=f(x)
$$

with boundary conditions

$$
u(0)=u(1)=0, \text { and } f(x)=2+\frac{1}{\Gamma(0.5)}\left(2.65 x^{1.5}-2 x^{0.5}\right),
$$

${ }_{0}^{c} D_{x}^{0.5}$ is the Caputo fractional derivative operator.

The exact solution of this problem is, $u(x)=x(x-1)$.

Solving this FBVP by the proposed method in section 3, we find the approximate results, absolute errors using first 5 Modified Legendre polynomials, Bernoulli polynomials, and Bernstein polynomials. We compare our accuracy of the absolute errors versus the errors obtained by the SincGalerkin method [5] with $L=5, M=5$, are shown in Table 1. Also we compare the approximate results with the exact solutions by displaying in Figure 1. We note that though the Bernstein polynomials converges slowly but we may observe that the accuracy is acceptable.

Again, 
Table 1. Absolute errors obtained by present method using three types of polynomials for example 1 .

\begin{tabular}{|c|c|c|c|c|c|c|}
\hline \multirow{2}{*}{$\mathbf{x}$} & \multirow{2}{*}{$\begin{array}{l}\text { Exact } \\
\text { Solutions }\end{array}$} & \multicolumn{3}{|c|}{$\begin{array}{l}\text { Absolute errors obtained by present method using } 5 \\
\text { polynomials }\end{array}$} & \multirow{2}{*}{$\begin{array}{l}\text { Absolute errors using } 9 \\
\text { Bernstein polynomials }\end{array}$} & \multirow{2}{*}{$\begin{array}{l}\text { Sinc-Galerkin method } \\
\text { [5] with } L=5, M=5\end{array}$} \\
\hline & & Legendre & Bernoulli & Bernstein & & \\
\hline 0 & 0 & 0 & 0 & 0 & 0 & 0 \\
\hline 0.1 & -0.09000 & $1.30 \times 10^{-4}$ & $1.29 \times 10^{-4}$ & $2.36 \times 10^{-2}$ & $1.30 \times 10^{-4}$ & $7.79 \times 10^{-4}$ \\
\hline 0.2 & -0.16000 & $2.54 \times 10^{-4}$ & $2.51 \times 10^{-4}$ & $3.51 \times 10^{-2}$ & $2.54 \times 10^{-4}$ & $2.34 \times 10^{-3}$ \\
\hline 0.3 & -0.21000 & $3.62 \times 10^{-4}$ & $3.60 \times 10^{-4}$ & $6.07 \times 10^{-2}$ & $3.62 \times 10^{-4}$ & $1.74 \times 10^{-3}$ \\
\hline 0.4 & -0.24000 & $4.48 \times 10^{-4}$ & $4.44 \times 10^{-4}$ & $8.10 \times 10^{-2}$ & $4.48 \times 10^{-4}$ & $4.25 \times 10^{-4}$ \\
\hline 0.5 & -0.25000 & $5.03 \times 10^{-4}$ & $4.98 \times 10^{-4}$ & $8.59 \times 10^{-2}$ & $5.03 \times 10^{-4}$ & $1.72 \times 10^{-3}$ \\
\hline 0.6 & -0.24000 & $5.18 \times 10^{-4}$ & $5.13 \times 10^{-4}$ & $1.01 \times 10^{-1}$ & $5.18 \times 10^{-4}$ & $1.19 \times 10^{-3}$ \\
\hline 0.7 & -0.21000 & $4.48 \times 10^{-4}$ & $4.79 \times 10^{-4}$ & $1.31 \times 10^{-1}$ & $4.84 \times 10^{-4}$ & $5.87 \times 10^{-4}$ \\
\hline 0.8 & -0.16000 & $3.93 \times 10^{-4}$ & $3.88 \times 10^{-4}$ & $1.37 \times 10^{-1}$ & $3.93 \times 10^{-4}$ & $1.55 \times 10^{-3}$ \\
\hline 0.9 & -0.09000 & $2.34 \times 10^{-4}$ & $2.31 \times 10^{-4}$ & $8.86 \times 10^{-2}$ & $2.34 \times 10^{-4}$ & $4.46 \times 10^{-4}$ \\
\hline 1 & 0 & 0 & 0 & 0 & 0 & 0 \\
\hline
\end{tabular}

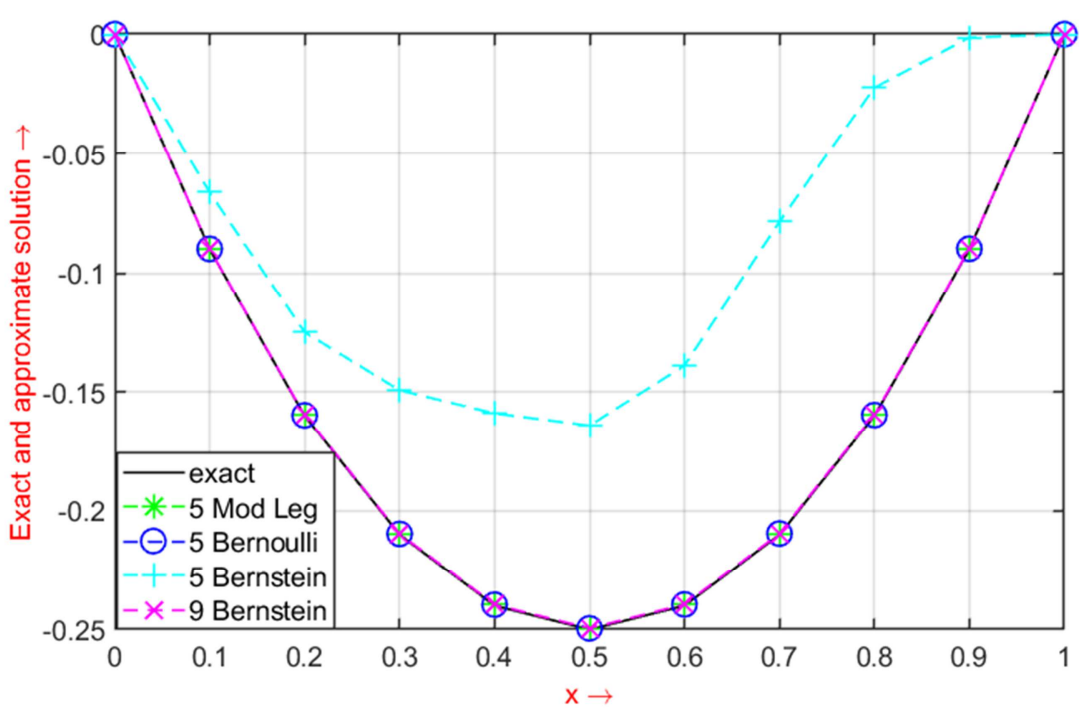

Figure 1. Exact and approximate solutions of Example 1.

Example 2. Consider the linear fractional boundary value problem $[5,7]$

$$
u^{\prime \prime}+0.5_{0}^{c} D_{x}^{0.3} u(x)+u(x)=f(x),
$$

with the boundary conditions

$$
u(0)=u(1)=0, \text { and }
$$

$f(x)=4 x^{2}(5 x-3)+0.5 x^{3.7}\left(\frac{120}{\Gamma(5.7)} x-\frac{24}{\Gamma(4.7)}\right)+x^{4}(x-1)$.

The exact solution of this problem is $u(x)=x^{4}(x-1)$.

In this case, we compute the absolute errors using first 5 polynomials of each type: Modified Legendre, Bernoulli, and Bernstein. We compare the accuracy of the absolute errors versus the errors obtained by the Sinc-Galerkin method [5] with $L=5, M=5$, and also with the Cubic Spline method [7], are shown in Table 2. The approximate results and the exact solutions are displayed in Figure 2. In this case, the present method is reliable with good accuracy.

Example 3. Consider the linear fractional boundary value problem $[5,7]$

$$
u^{\prime \prime}-x u^{\prime}(x)+{ }_{0}^{c} D_{x}^{0.5} u(x)=f(x),
$$

with the boundary conditions

$$
u(0)=u(1)=0 \text {, and }
$$

$$
f(x)=-3 x^{3}-2 x^{2}+8 x+2+\frac{1}{\Gamma(0.5)}\left(2.67 x^{1.5}+3.2 x^{2.5}-4 x^{0.5}\right)
$$

The exact solution of this problem is $u(x)=x^{2}(x+1)-2 x$.

Table 2. Absolute errors obtained by present method using three types of polynomials for example 2.

\begin{tabular}{lllllll}
\hline \multirow{2}{*}{$\mathbf{x}$} & $\begin{array}{l}\text { Exact } \\
\text { Solutions. }\end{array}$ & \multicolumn{2}{l}{ Absolute errors obtained by present method using 5 polynomials } & $\begin{array}{l}\text { Sinc-Galerkin method } \\
\text { [5] with } \boldsymbol{L}=\mathbf{5}, \boldsymbol{M}=\mathbf{5}\end{array}$ & $\begin{array}{l}\text { Cubic Spline } \\
\text { method [7] }\end{array}$ \\
\cline { 3 - 5 } & Legendre & Bernoulli & Bernstein & 0 & \\
0 & 0 & 0 & 0 & 0 & 0 & 0 \\
0.125 & -0.00021 & $1.73 \times 10^{-4}$ & $1.13 \times 10^{-4}$ & $1.81 \times 10^{-4}$ & $2.80 \times 10^{-4}$ & $2.00 \times 10^{-3}$ \\
\hline
\end{tabular}




\begin{tabular}{lllllll}
\hline \multirow{2}{*}{$\mathbf{x}$} & $\begin{array}{l}\text { Exact } \\
\text { Solutions. }\end{array}$ & \multicolumn{2}{l}{ Absolute errors obtained by present method using 5 polynomials } & $\begin{array}{l}\text { Sinc-Galerkin method } \\
\text { [5] with } \boldsymbol{L}=\mathbf{5}, \boldsymbol{M}=\mathbf{5}\end{array}$ & $\begin{array}{l}\text { Cubic Spline } \\
\text { method [7] }\end{array}$ \\
\cline { 3 - 6 } & Legendre & Bernoulli & Bernstein & $4.72 \times 10^{-3}$ & $4.08 \times 10^{-3}$ \\
0.250 & -0.00292 & $3.38 \times 10^{-4}$ & $2.22 \times 10^{-4}$ & $1.73 \times 10^{-3}$ & $4.28 \times 10^{-3}$ & $5.83 \times 10^{-3}$ \\
0.375 & -0.01235 & $4.82 \times 10^{-4}$ & $3.12 \times 10^{-4}$ & $4.52 \times 10^{-3}$ & $3.00 \times 10^{-3}$ & $6.85 \times 10^{-3}$ \\
0.500 & -0.03125 & $5.77 \times 10^{-4}$ & $3.59 \times 10^{-4}$ & $6.92 \times 10^{-3}$ & $3.17 \times 10^{-3}$ & $6.81 \times 10^{-3}$ \\
0.625 & -0.05722 & $5.86 \times 10^{-4}$ & $3.33 \times 10^{-4}$ & $8.86 \times 10^{-3}$ & $1.85 \times 10^{-3}$ & $5.57 \times 10^{-3}$ \\
0.750 & -0.07910 & $4.77 \times 10^{-4}$ & $2.17 \times 10^{-4}$ & $1.06 \times 10^{-2}$ & $3.09 \times 10^{-3}$ & $3.26 \times 10^{-3}$ \\
0.875 & -0.07327 & $2.55 \times 10^{-4}$ & $5.31 \times 10^{-5}$ & $9.86 \times 10^{-3}$ & 0 & 0 \\
1 & 0 & 0 & 0 & 0 & & 0 \\
\hline
\end{tabular}

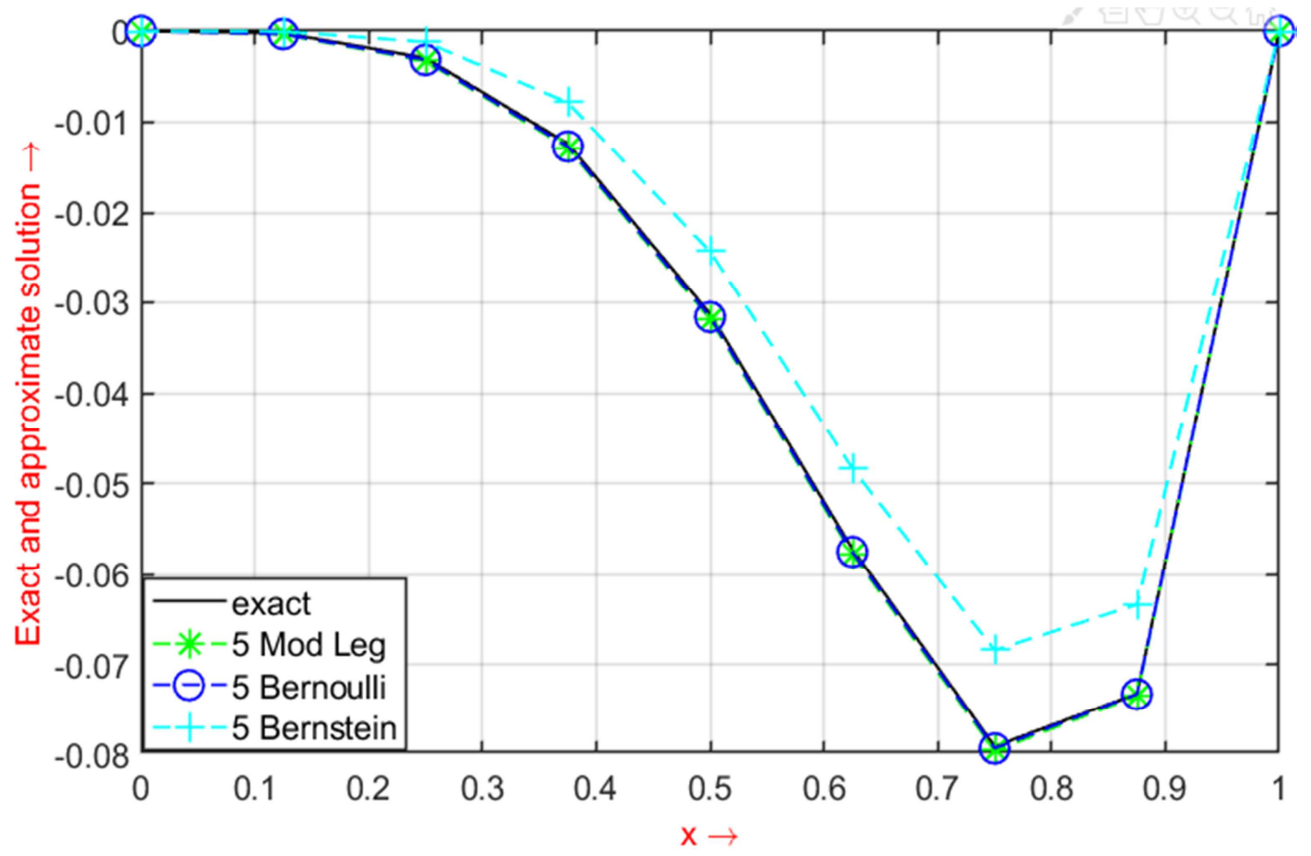

Figure 2. Exact and approximate solutions of Example 2.

In this problem, the absolute errors using first 5 polynomials of each type: Modified Legendre, Bernoulli, and Bernstein, and first 9 Bernstein polynomials are computed. We compare the accuracy of the absolute errors versus the errors obtained by the Sinc-Galerkin method [5] with
$L=5, M=5$, and are shown in Table 3.

The approximate results and the exact solutions are displayed in Figure 3. In this case, the accuracy is found with good agreement compare to the solutions of the existing method and exact solutions.

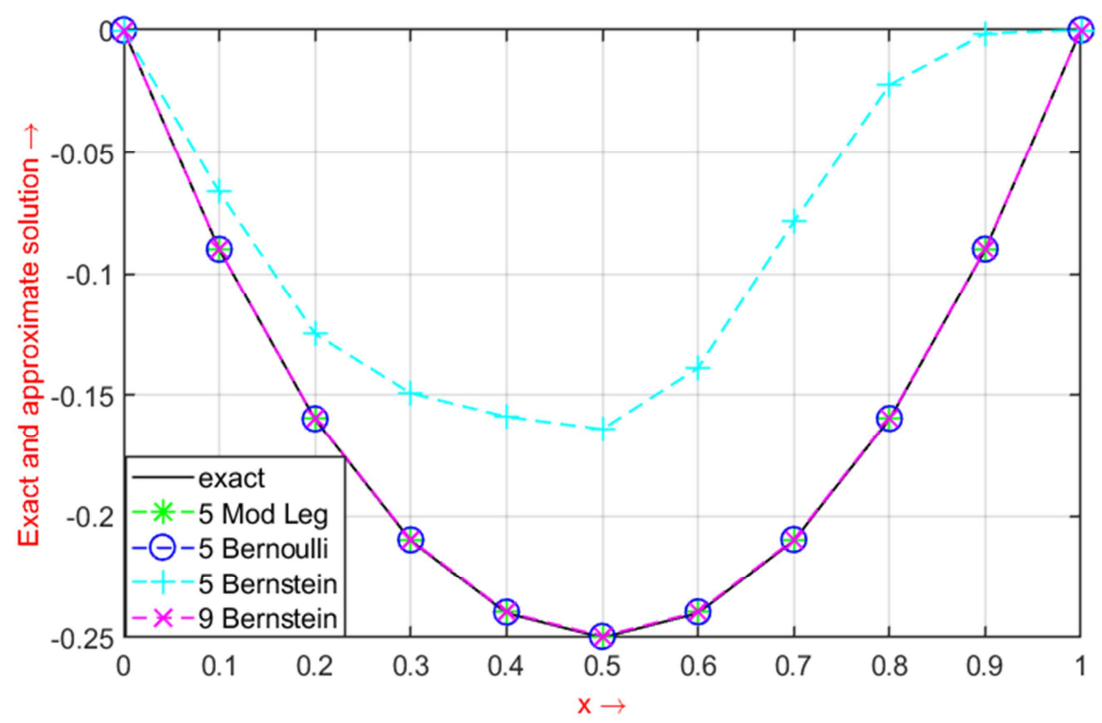

Figure 3. Exact and approximate solutions of Example 3. 
Table 3. Absolute errors obtained by present method using three types of polynomials for example 3.

\begin{tabular}{|c|c|c|c|c|c|c|}
\hline \multirow{2}{*}{$\mathbf{x}$} & \multirow{2}{*}{$\begin{array}{l}\text { Exact } \\
\text { Solutions }\end{array}$} & \multicolumn{3}{|c|}{ Absolute errors obtained by present method using 5 polynomials } & \multirow{2}{*}{$\begin{array}{l}\text { Absolute Errors using } 9 \\
\text { Bernstein Polynomials }\end{array}$} & \multirow{2}{*}{$\begin{array}{l}\text { Sinc-Galerkin method } \\
\text { [5] with } L=5, M=5\end{array}$} \\
\hline & & Legendre & Bernoulli & Bernstein & & \\
\hline 0 & 0 & 0 & 0 & 0 & 0 & 0 \\
\hline 0.1 & -0.18900 & $2.59 \times 10^{-5}$ & $2.61 \times 10^{-5}$ & $1.04 \times 10^{-1}$ & $5.39 \times 10^{-3}$ & $4.32 \times 10^{-5}$ \\
\hline 0.2 & -0.35200 & $5.02 \times 10^{-5}$ & $5.09 \times 10^{-5}$ & $1.32 \times 10^{-1}$ & $5.54 \times 10^{-3}$ & $8.58 \times 10^{-5}$ \\
\hline 0.3 & -0.48300 & $7.25 \times 10^{-5}$ & $7.30 \times 10^{-5}$ & $2.04 \times 10^{-1}$ & $8.35 \times 10^{-4}$ & $1.30 \times 10^{-4}$ \\
\hline 0.4 & -0.57600 & $9.07 \times 10^{-5}$ & $9.09 \times 10^{-5}$ & $2.35 \times 10^{-1}$ & $4.17 \times 10^{-3}$ & $1.69 \times 10^{-4}$ \\
\hline 0.5 & -0.62500 & $1.03 \times 10^{-4}$ & $1.03 \times 10^{-4}$ & $2.40 \times 10^{-1}$ & $6.65 \times 10^{-3}$ & $2.01 \times 10^{-4}$ \\
\hline 0.6 & -0.62400 & $1.08 \times 10^{-4}$ & $1.07 \times 10^{-4}$ & $2.95 \times 10^{-1}$ & $6.71 \times 10^{-3}$ & $2.24 \times 10^{-4}$ \\
\hline 0.7 & -0.56699 & $1.02 \times 10^{-4}$ & $1.02 \times 10^{-4}$ & $3.85 \times 10^{-1}$ & $5.52 \times 10^{-3}$ & $2.48 \times 10^{-4}$ \\
\hline 0.8 & -0.44799 & $8.53 \times 10^{-5}$ & $8.51 \times 10^{-5}$ & $3.97 \times 10^{-1}$ & $3.84 \times 10^{-3}$ & $2.40 \times 10^{-4}$ \\
\hline 0.9 & -0.26100 & $5.21 \times 10^{-5}$ & $5.20 \times 10^{-5}$ & $2.58 \times 10^{-1}$ & $1.99 \times 10^{-3}$ & $1.61 \times 10^{-4}$ \\
\hline 1 & 0 & 0 & 0 & 0 & 0 & 0 \\
\hline
\end{tabular}

\section{Conclusion}

In this paper, three polynomials such as Legendre, Bernstein, and Bernoulli polynomials have been exploited for solving linear fractional order two-point boundary value problems by the well-known Galerkin method. The proposed formulation is easy to understand and can be coded easily. The approximate results converge monotonically to the exact solutions in each cases. In addition, fast convergence has been achieved in some cases even with a few polynomials of lower order. Finally, we may conclude that this method may be applied any order of linear and nonlinear fractional ordinary or partial differential equations with specified boundary conditions.

\section{References}

[1] I. Podlubny, "Fractional Differential Equations: An Introduction to Fractional Derivatives, Fractional Differential Equations, to Methods of Their Solution and Some of Their Applications," vol. 198, Academic Press, San Diego, USA, 1999.

[2] M. Delkhosh, "Introduction of derivatives and integrals of fractional order and its applications," Applied Mathematics and Physics, 1 (2013), 103-119.

[3] S. Momani and Z Odibat, "Numerical comparison of methods for solving linear differential equations of fractional order," Chaos, Solutions and Fractals, 31 (2007), 1248-1255.

[4] Jin-Fa Cheng and Yu-Ming Chu, "Solution to the linear fractional differential equation using Adomian decomposition method," Mathematical Problems in Engineering, Vol. 2011, Article ID 587068, 14 pages, doi: 10.1155/2011/587068.

[5] A. Secer, S. Alkan, M. A. Akinlar and M. Bayram, "SincGalerkin method for approximate solutions of fractional order boundary value problems," Boundary value problems, 2013: 281, 2013.

[6] Z. Odibat, S. Momani, V. S. Erturk, "Generalized differential transform method: Application to differential equations of fractional order," Applied Mathematics and Computation, 197 (2008), 467-477.

[7] W. K. Zahra and S. M. Elkholy, "Cubic spline solution of fractional Bagley-Torvik equation, Electronic Journal of Mathematical Analysis and Applications, 1 (2013), 230-241.

[8] Xinxiu Li, "Numerical solution of fractional differential equations using cubic B-Spline wavelet collocation method," Commun Nonlinear Sci Numer Simulat, 17 (2012) 39343946.

[9] H. Jafari, S. A. Yousefi, M. A. Firoozjaee, S. Momani, C. M. Khalique, "Application of Legendre wavelets for solving fractional differential equations," Computers and Mathematics with Applications, 62 (2011), 1038-1045.

[10] Qasem M. Al-Mdallal, M. Syam and M. N. Anwar, "A collocation-shooting method for solving fractional boundary value problems," Commun Nonlinear Sci Numer Simulat, 15 (2010), 3814-3822.

[11] E. Demirci and N. Ozalp, "A method for solving differential equations of fractional order", Journal of Computational and Applied Mathematics, 236 (2012), 2754-2762).

[12] T. Zhang and C. Tong, "A remark on the fractional order differential equations," Journal of Computational and Applied Mathematics, 340 (2018), 375-379.

[13] F. Mohammadi and C. Cattani, "A generalized fractional-order Legendre wavelet Tau method for solving fractional differential equations," Journal of Computational and Applied Mathematics, 339 (2018), 306-316.

[14] P. E. Lewis and J. P. Ward, "The Finite Element Method, Principles and Applications," Addison-Wesley, 1991.

[15] M. S. Islam and M. B. Hossain, "Numerical solutions of eighth order BVP by the Galerkin residual technique with Bernstein and Legendre polynomials", Applied Mathematics and Computation, 261 (2015), 48-59.

[16] M. S. Islam and A. Shirin, "Numerical solutions of a class of second order boundary value problems on using Bernoulli Polynomials," Applied Mathematics, 2 (2011), 1059-1067. 